\title{
The effects of short-term high-fat feeding on exercise capacity: multi-tissue transcriptome changes by RNA sequencing analysis
}

Ya Xiao ${ }^{1,2+}$, Wanshan Wang ${ }^{3 \dagger}$, Liguo Chen ${ }^{1 \dagger}$, Jieyu Chen ${ }^{2}$, Pingping Jiang ${ }^{2}$, Xiuqiong Fu ${ }^{4}$, Xiaoli Nie ${ }^{2}$, Hiuyee Kwan ${ }^{4}$, Yanyan Liu' ${ }^{2^{*}}$ and Xiaoshan Zhao ${ }^{2^{*}}$

\begin{abstract}
Background: The effects of short-term high fat diets on physiology are elusive and the molecular changes following fat overconsumption remain largely unknown. In this study, we aimed to evaluate exercise capacity in mice fed with a high fat diet (HFD) for 3 days and investigate the molecular mechanisms in the early response to high-fat feeding.

Methods: Exercise capacity was assessed by weight-loaded swimming test in mice fed a control diet (10 kcal\% fat) or a HFD (60 kcal\% fat) for 3 days. Global gene expression of ten important tissues (brain, heart, liver, spleen, lung, kidney, stomach, duodenum, skeletal muscle and blood) was analyzed using RNA Sequencing.

Results: A HFD for just 3 days can induce $71 \%$ decrease of exercise performance prior to substantial weight gain $(P<0$. 01). Principle component analysis revealed that differential gene expression patterns existed in the ten tissues. Out of which, the brain, spleen and lung were demonstrated to have more pronounced transcriptional changes than other tissues. Biological process analysis for differentially expressed genes in the brain, spleen and lung showed that dysregulation of peripheral and central immune response had been implicated in the early stage of HFD exposure. Neurotransmission related genes and circulatory system process related genes were significantly down-regulated in the brain and lung, respectively.
\end{abstract}

Conclusions: Our findings provide new insights for the deleterious effects of high-fat feeding, especially revealing that the lung maybe as a new important target attacked by short-term high-fat feeding.

Keywords: RNA Sequencing, High-fat feeding, Exercise capacity, Multi-tissue, Short-term

\section{Background}

High-fat and high-calorie diets along with a low physical activity lifestyle have contributed to the onset or development of type 2 diabetes, metabolic syndrome and cardiovascular disease [1]. There has been conflicting results on whether consumption of a high fat diet (HFD) is detrimental or beneficial for endurance performance. Studies in rats have demonstrated a beneficial effect of a fat-rich diet on exercise capacity via increasing the

\footnotetext{
*Correspondence: siyecao2015@163.com; zhaoxs0609@163.com

${ }^{\dagger}$ Equal contributors

${ }^{2}$ School of Traditional Chinese Medicine, Southern Medical University,

Guangzhou, China

Full list of author information is available at the end of the article
}

ability to oxidize fat and concomitantly sparing glycogen content [2-4]. In contrast, Murray et al. [5] reported that 9 days of high-fat feeding impaired energy production and physical performance associated with respiratory uncoupling in skeletal muscle mitochondria. In the present study, we aimed to evaluate exercise capacity in mice fed with a HFD for 3 days and investigate the molecular mechanisms in the early response to high-fat feeding.

Advances in genomic technologies may help to reveal the early molecular changes by enabling simultaneous analysis of thousands of genes in response to a HFD. The serial analysis of gene expression strategy identified 12 transcripts of hypothalamus which regulated by food intake in mice at $3 \mathrm{~h}$ after high-fat meal ingestion [6]. 
The transcriptomic analysis of duodenum mucosa after high-fat meal ingestion in C57BL/6 J mice found substantial changes of genes related to lipid metabolism [7]. Microarray analysis showed markedly changes of numerous genes involved in various biological processes including morphogenesis, fatty acid catabolism and amino acid metabolism following 3 days of high-fat feeding in the skeletal muscle of C57BL/6 J mice [8]. cDNA microarrays analysis of mRNA expression showed downregulation of genes related to fatty acid biosynthesis in the liver of one week HFD-fed BALB/c mice [9].

However, no study to date has simultaneously analyzed the systemic gene expression profile of multi-tissues in response to short-term HFD and it remains unknown that which tissue has the most pronounced changes of gene expression profile in the early stage after high-fat feeding. Recently, RNA sequencing (RNA-seq), as an attractive alternative to microarrays for transcriptome analysis, provides major advances in robustness, comparability and richness of expression profiling data [10]. Thus we utilized RNA-seq to investigate gene expression profile of ten tissues (brain, heart, liver, spleen, lung, kidney, stomach, duodenum, skeletal muscle and blood) in C57BL/6 J mice with 3 days of high-fat feeding, which may contribute to the understanding of molecular mechanisms of changes in exercise performance induced by short-term HFD.

\section{Methods}

\section{Animals and study protocol}

Animal experiments were approved by the Animal Care and Use Committee of Southern Medical University (Approval No.2013027). The methods were carried out in accordance with the approved guidelines. Forty male C57BL/6 J mice at the age of 8 weeks were obtained from Laboratory Animal Center of Southern Medical University (Approval No. SCXK (Yue) 2011-0015). All the animals were maintained in a temperaturecontrolled room $\left(22-25{ }^{\circ} \mathrm{C}\right.$; $35-55 \%$ humidity) with a twelve-hour light/dark cycle. Mice were randomly divided into two groups, where 20 mice were fed a control diet (CD, D12450B, $10 \mathrm{kcal} \%$ fat) and 20 mice were fed a high fat diet (HFD, D12492, $60 \mathrm{kcal} \%$ fat) for 3 days. Mice were allowed free access to food and water. The changes of body weight were observed after 3 days.

\section{Assessment of exercise capacity}

A weight-loaded swimming test has been commonly used for assessment of exercise capacity in murine [11, 12]. After 3 days, 10 mice were taken out from each group for swimming exercise performance test which was conducted as previously described with some modifications [13]. The mice were not fasted and were loaded the constant weight ( $1.5 \mathrm{~g}$ tin wire, attached to the tail). The mice were dropped individually into a swimming pool $(30 \mathrm{~cm}$ high, $25 \mathrm{~cm}$ in diameter) filled with water at $25 \pm 1{ }^{\circ} \mathrm{C}$. It was considered that the mice were exhausted when they failed to return to the surface of water within a $10 \mathrm{~s}$ period. The swimming time to exhaustion was used as the index of exercise capacity.

\section{Biochemical assays}

After 3 days of feeding, the remaining 10 mice in each group were anesthetized with sodium pentobarbital (75 $\mathrm{mg} / \mathrm{kg}$, ip) following a $6 \mathrm{~h}$ fasting period. The blood samples were collected by removing the left eyeball of the mice and rapidly centrifuged at $1000 \mathrm{~g}$ at $4{ }^{\circ} \mathrm{C}$ for $10 \mathrm{~min}$. Plasma levels of glucose, triglycerides, total cholesterol, low-density lipoprotein-cholesterol (LDL-C), high-density lipoprotein-cholesterol (HDL-C), free fatty acids (FFAs), apolipoprotein $\mathrm{E}$ (ApoE), C-reactive protein (CRP), superoxide dismutase (SOD), homocysteine (HCY), alanine aminotransferase (ALT), aspartate aminotransferase (AST), alkaline phosphatase (ALP), total protein (TP), albumin (ALB), globulin (GLB), ALB/GLB, total bilirubin (TBIL), direct bilirubin (DBIL), indirect bilirubin (IBIL), total bile acid (TBA), uric acid (UA), Creatinine $(\mathrm{Cr})$, urea, Cystatin $\mathrm{C}(\mathrm{Cys} \mathrm{C})$, creatine kinase (CK), lactate dehydrogenase (LDH), A-hydroxybutyric acid dehydrogenase ( $\mathrm{HBDH})$, potassium $(\mathrm{K})$, sodium $(\mathrm{Na})$, chlorine $(\mathrm{Cl})$ and calcium $(\mathrm{Ca})$ were measured using a multifunctional biochemistry analyzer (Olympus AU2700, Tokyo, Japan). Statistical analyses for biochemical assays and assessment of exercise capacity were conducted using SPSS (version 19.0) for Windows. The data are reported as mean \pm standard error of the mean (SEM). Differences between the compared groups were analyzed by Student's $t$ test. A $P$ value less than 0.05 was considered to be statistically significant.

\section{Tissue Processing and RNA Isolation}

Five mice of each group were selected randomly from the mice which did not perform weight-loaded swimming test for RNA sequencing. Tissue samples included the whole brain and heart, liver, spleen, lung, kidney, stomach, duodenum, skeletal muscle and blood. The samples were dissected and immediately immersed in RNA later solution (Ambion, California, USA). All samples were stored at $-80{ }^{\circ} \mathrm{C}$ before processing. Total RNA was extracted from all samples using Trizol reagent (Invitrogen, Carlsbad, CA). The RNA concentration was quantified using a spectrophotometer (NanoDrop echnologies, Wilmington, DE) and the integrity was evaluated by the Agilent Bioanalyzer 2100 (Agilent, Santa Clara, CA).

\section{RNA sequencing and gene expression analysis}

In the CD and HFD group, fixed quantities of RNA of five samples from the same kind of tissue were 
combined into a single sample. The cDNA library was conducted by Illumina Tru-Seq RNA Sample Prep Kits (Illumina, San Diego, CA) with Ribosomal RNA depletion following manufacturer's instructions. Samples were sequenced for 50 bp single read using the HiSeq2000 platform. Before alignment, reads with a low quality and adapters were screened by FastQC and removed. The remaining reads were mapped to the mice reference genome (UCSC mm10) with TopHat v2.0.9. The maximum number allowed for mismatch mapping was 2 . Reads Per Kilobase of exon model per Million mapped reads (RPKM) was calculated to express the mRNA abundances. Analysis of differential expression was performed using edgeR, which could be used even with the most minimal levels of replication [14]. The read counts per gene were normalized to counts per million (CPM). CPM values were utilized for differential expression analysis, whereas RPKM values were used for principle component analysis (PCA) with the GeneSpring Gx 12.0 software (Agilent Technologies, Palo Alto, CA).

\section{Biological process analysis}

Molecule annotation system (MAS) is a set of web tools for function annotation based on integration of various public resources such as Gene Ontology, KEGG, BioCarta, GenMapp, UniGene, OMIM and more [15]. Biological process analysis for differentially expressed genes (DEGs) was performed using the CapitalBio MAS 3.0 software (CapitalBio Corporation, Beijing, China). Absolute fold change $>2$ with $P<0.05$ was considered statistically significant in the RNA-seq analysis.

\section{Real-time quantitative RT-PCR verification of RNA-seq data}

To further confirm the findings from the RNA-seq analysis, we selectively examined 22 genes expression $(8$ genes in the brain, 9 genes in the spleen and 5 genes in the lung) using real-time quantitative RT-PCR (qRTPCR) method. Five samples from the same kind of tissue of purified RNA in each group were used for qRT-PCR. Total RNA from the samples was first reverse- transcribed into cDNA templates with the PrimeScriptTM RT reagent Kit (TaKaRa, Otsu, Japan) according to the manufacturer's instruction. PCR was run on a ABI 7500 Real-Time PCR System (Applied Biosystems, Inc., Foster City, CA, USA) using the SYBR Premix Ex TaqTM II (Otsu-Shi, Shiga, Japan). The reaction volume was $20 \mu \mathrm{L}$ and the PCR conditions were as follows: $30 \mathrm{~s}$. at $95{ }^{\circ} \mathrm{C}$, 40 cycles of $5 \mathrm{~s}$. at $95{ }^{\circ} \mathrm{C}$ and $34 \mathrm{~s}$. at $60^{\circ} \mathrm{C}$, followed by a melting curve analysis step. Every sample was measured in duplicate, and relative quantification was determined by the comparative $\mathrm{Ct}$ method $(2-\Delta \Delta \mathrm{CT})$. $\beta$-actin was used as a housekeeping gene to normalize the expression data. The primers used for gene validation are listed in Additional file 1: Table S1.

\section{Results \\ Body weight and blood plasma metabolites}

As shown in Table 1, initial and final body weights showed no significantly differences among the groups. HFD feeding tended to increase weight gain, but this effect failed to reach statistical significance $(P=0.094)$. Plasma glucose levels were $67 \%$ higher in HFD-fed mice than CD-fed mice $(P<0.001)$. Although no differences were seen in plasma LDL-C, FFA and ApoE, the cholesterol level was significantly greater in the HFD-fed mice $(P<0.001)$. The increase in HDL-C $(P<0.001)$ and decrease in plasma triglycerides $(P=0.005)$ were also observed in HFD-fed mice. Plasma levels of CRP, SOD, HCY, ALT, AST, ALP, TP, ALB, GLB, ALB/GLB, TBIL, DBIL, IBIL, TBA, UA, Cr, Urea, $\mathrm{Cys} C, \mathrm{LDH}, \mathrm{HBDH}, \mathrm{K}$ and $\mathrm{Ca}$ were unchanged between the CD and HFD groups except for the CK, Na and $\mathrm{Cl}$ (Additional file 2: Table S2).

\section{Exercise capacity in a weight-loaded swimming test}

The swimming time indicated the exercise capacity. Both groups of mice swam the same mean time at baseline $(\mathrm{CD}=687.3 \pm 93.9 \mathrm{~s}, \mathrm{HFD}=669.0 \pm 87.8 \mathrm{~s})$. After 3 days of feeding, CD-fed mice maintained a similar swimming time of $748.9 \pm 77.4 \mathrm{~s}$, whereas HFD-fed mice swam $213.7 \pm 44.4 \mathrm{~s}$ on average, $71 \%$ less far than the CD-fed mice $(P<0.001)$ (Fig. 1$)$.

\section{Summary of sequencing data and global gene expression profiles}

A range of 28.1 to 67.4 million raw reads were generated among samples. After removing reads with a low quality, an average of 32.3 million clean reads per sample was obtained (range, 21.3 to 51.5 million reads). Approximately $98.64 \%$ of clean reads per sample were mapped to the mice reference genome among samples (Table 2).

Table 1 Body weight and plasma biochemical parameters of CD-fed and HFD-fed mice

\begin{tabular}{lrll}
\hline Parameter & \multicolumn{1}{l}{ CD } & \multicolumn{1}{l}{ HFD } & $P$ value \\
\hline Initial body weight $(\mathrm{g})$ & $22.81 \pm 0.29$ & $22.84 \pm 0.31$ & 0.950 \\
Final body weight $(\mathrm{g})$ & $22.64 \pm 0.40$ & $23.45 \pm 0.26$ & 0.094 \\
Plasma glucose $(\mathrm{mmol} / \mathrm{L})$ & $4.71 \pm 0.42$ & $7.89 \pm 0.31$ & $<.001$ \\
Plasma triglycerides $(\mathrm{mmol} / \mathrm{L})$ & $0.46 \pm 0.03$ & $0.36 \pm 0.01$ & 0.005 \\
Plasma cholesterol (mmol/L) & $2.16 \pm 0.08$ & $3.25 \pm 0.08$ & $<.001$ \\
Plasma LDL-C (mmol/L) & $0.13 \pm 0.02$ & $0.19 \pm 0.04$ & 0.233 \\
Plasma HDL-C (mmol/L) & $1.55 \pm 0.05$ & $2.30 \pm 0.04$ & $<.001$ \\
Plasma FFA (mmol/L) & $0.85 \pm 0.04$ & $0.88 \pm 0.04$ & 0.611 \\
Plasma ApoE (mg/L) & $16.34 \pm 3.24$ & $21.30 \pm 2.57$ & 0.254 \\
\hline
\end{tabular}

Values are expressed as means \pm SEM. CD, control diet; HFD high fat diet 


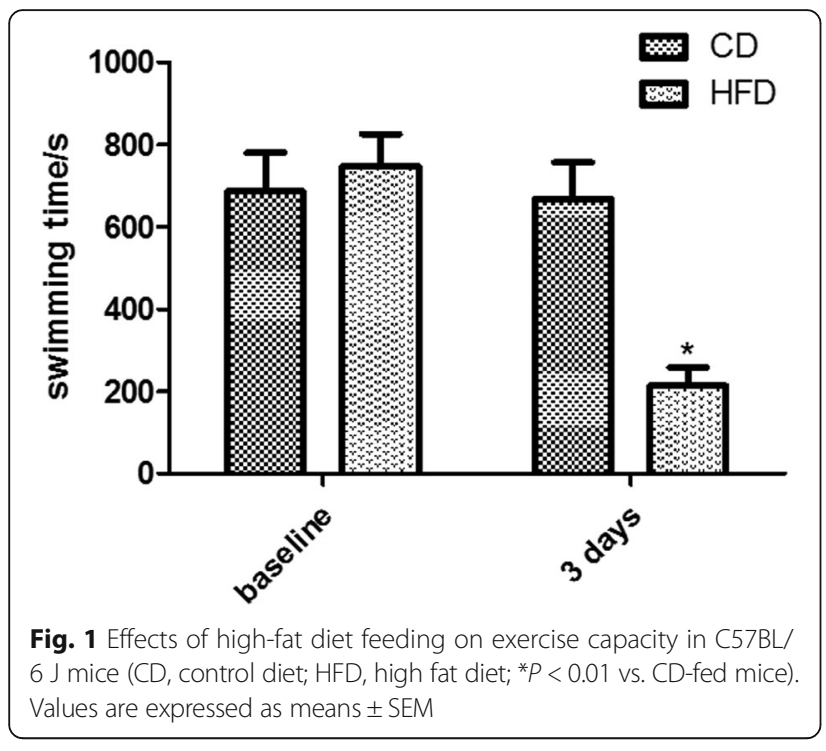

Totally 33151 unique genes among all samples was detected. To assess the effect of sequencing depth on RNA-seq data, we conducted sequencing saturation analysis. In the beginning of the RNA-seq, with increase of the counts of reads, the number of identified genes in

Table 2 Summary of sequence statistics

\begin{tabular}{lllll}
\hline Sample & $\begin{array}{l}\text { Total number } \\
\text { of raw reads }\end{array}$ & $\begin{array}{l}\text { Total number of } \\
\text { clean reads }\end{array}$ & $\begin{array}{l}\text { Mapped } \\
\text { reads }\end{array}$ & $\begin{array}{l}\text { Mapping } \\
\text { ratio }(\%)\end{array}$ \\
\hline C-blood & $38,700,424$ & $30,614,380$ & $30,228,909$ & $98.74 \%$ \\
C-brain & $52,264,883$ & $40,188,570$ & $39,152,260$ & $97.42 \%$ \\
C- & $59,741,001$ & $45,938,467$ & $45,365,742$ & $98.75 \%$ \\
duodenum & & & & \\
C-heart & $30,948,992$ & $23,609,651$ & $23,392,762$ & $99.08 \%$ \\
C-kidney & $52,803,870$ & $40,421,377$ & $40,024,878$ & $99.02 \%$ \\
C-liver & $36,944,726$ & $28,422,843$ & $28,121,458$ & $98.94 \%$ \\
C-lung & $28,118,618$ & $21,811,801$ & $21,603,740$ & $99.05 \%$ \\
C-muscle & $38,907,555$ & $29,713,640$ & $29,393,124$ & $98.92 \%$ \\
C-spleen & $46,764,891$ & $36,109,723$ & $34,944,640$ & $96.77 \%$ \\
C-stomach & $45,416,694$ & $34,795,951$ & $34,206,646$ & $98.31 \%$ \\
H-blood & $58,119,027$ & $46,530,915$ & $46,004,745$ & $98.87 \%$ \\
H-brain & $29,603,099$ & $22,773,050$ & $22,369,746$ & $98.23 \%$ \\
H- & $49,387,591$ & $37,878,983$ & $37,445,186$ & $98.85 \%$ \\
duodenum & & & & \\
H-heart & $38,642,015$ & $29,564,572$ & $29,298,974$ & $99.10 \%$ \\
H-liver & $67,430,163$ & $51,556,269$ & $51,032,926$ & $98.98 \%$ \\
H-lung & $32,460,683$ & $25,169,611$ & $24,916,857$ & $99.00 \%$ \\
H-muscle & $30,898,190$ & $23,377,884$ & $23,142,897$ & $98.99 \%$ \\
H-spleen & $31,165,867$ & $24,237,199$ & $23,688,869$ & $97.74 \%$ \\
H-stomach & $27,849,155$ & $21,301,788$ & $21,072,130$ & $98.92 \%$ \\
H-kidney & $43,190,796$ & $33,268,647$ & $32,945,285$ & $99.03 \%$ \\
\hline C contro & $50 p, H n g h$ & 0 & &
\end{tabular}

$\mathrm{C}$ control diet group, $H$ high fat diet group each tissue was increasing. However, when the counts of reads rose to approximately 30 million, the growth rate of identified genes flattened which indicated that the number of identified genes tended to saturation.

To investigate the global gene expression profiles of ten tissues and identify the tissue with the most pronounced transcriptional changes after short-term highfat feeding, we performed PCA on all samples (Fig. 2). The results showed differential gene expression patterns in the ten tissues. Each sphere represented an individual sample. The sphere representing liver, heart, kidney, skeletal muscle and blood in CD and HFD group overlapped, indicating that gene expression patterns of HFDfed mice in the five tissues were almost not changed. The sphere representing stomach and duodenum in $\mathrm{CD}$ and HFD group were closely to each other, indicating that gene expression patterns of CD-fed and HFD-fed mice in the two tissues were nearly similar. Gene expression patterns of brain, spleen and lung in HFD group showed substantial differences as compared with CD group.

\section{Genes and the related biological processes altered in the brain of HFD-fed mice}

According to the results of PCA, we found that the brain, spleen and lung had more pronounced transcriptional changes than other tissues following 3 days of HFD intervention. Consequently, we focused on the analysis of the genes and related biological processes altered in the brain, spleen and lung of HFD-fed mice. We found 145 DEGs in the brain, of which less than half of the genes were annotated with known function from the Ensembl database (Table 3). To gain insight into the possible biologic functions of the genes affected by highfat feeding, enrichment analysis of Gene Ontology for the DEGs was conducted. After 3 days of HFD exposure, the overrepresented biological processes in the brain were mainly enriched in neurological system process and immune response (Fig. 3). In the neurological system process related group, LIM homeobox transcription factor 1 beta (Lmx1b), and NK2 homeobox 1(Nkx2-1), which involved in neuron migration and development, were down-regulated to 8.11-fold and 10.56-fold respectively in the HFD-fed mice. Genes related to central nervous system morphogenesis were significantly downregulated, such as homeobox D11 (Hoxd11) (12.21-fold) and UNC homeobox (Uncx) (5.39-fold). Inflammatory/ immune related processes were altered as well. The mRNA levels of chemokine (C-C motif) receptor $1(\mathrm{Ccr} 1)$ was up-regulated to 13.18 -fold. The rest immune-related genes including chemokine ( $\mathrm{C}-\mathrm{C}$ motif) receptor 4 (Ccr4), CD200 receptor 3 (Cd200r3), CD274 molecule (Cd274), CD300 antigen like family member G (Cd300lg) and transcription factor AP-2, alpha (Tfap2a) 


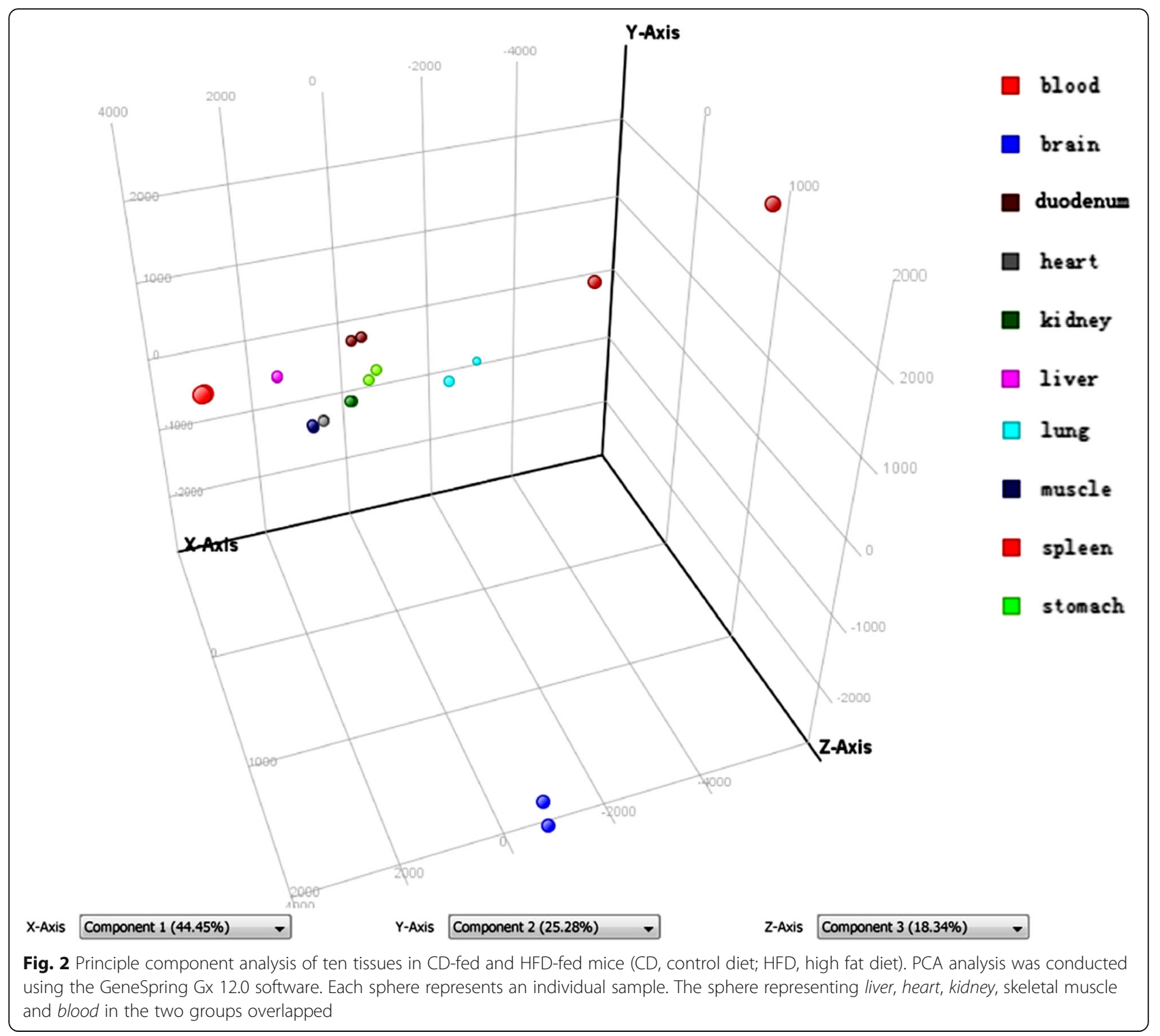

were down-regulated to 8.28-,3.92-, 3.48-, 11.96- and 4.79-fold respectively.

\section{Genes and the related biological processes altered in the spleen of HFD-fed mice}

61 genes were markedly changed in the spleen, however, half of which were largely unknown. The overrepresented biological processes in the spleen were mainly related to acute-phase response and immune system, with a significant change in the expression of immune-related genes (Fig. 3). As shown in Table 4, immunoglobulin kappa joining 4 (Igkj4) and T cell receptor alpha joining 37(Traj37) were increased to 8.34- and 70.52-fold respectively, while Fc receptor-like $S$, scavenger receptor (Fcrls), immunoglobulin heavy variable 1-84 (Ighv1-84), immunoglobulin kappa joining 1(Igkj1), immunoglobulin kappa variable 5-39 (Igkv5-39), regenerating isletderived 3 alpha (Reg3a) and regenerating islet-derived 3 beta (Reg3b) in various immunological pathways were decreased to 74.54-, 11.63-, 54.57-, 3.60-, 16.34- and 8.11- fold respectively. Other overrepresented biological processes included carbohydrate metabolism, ubiquitindependent protein catabolism, G2/M transition of mitotic cell cycle and neurotransmitter uptake.

Genes and the related biological processes altered in the lung of HFD-fed mice

In the lung, 83 genes were significantly altered. The overrepresented biological processes were mainly enriched in immune-related processes, including $\mathrm{T}$ cell and B cell mediated immune response (Fig. 3). As shown in Table 5, Immunoglobulin heavy constant epsilon 
Table 3 Differentially expressed genes in the brain of HFD-fed mice

\begin{tabular}{|c|c|c|c|c|c|c|c|c|}
\hline Gene symbol & $\log _{2} F C$ & $P$-value & Gene symbol & $\log _{2} \mathrm{FC}$ & $P$-value & Gene symbol & $\log _{2} \mathrm{FC}$ & $P$-value \\
\hline Alpi & -3.65 & 0.022 & Slc22a19 & -3.40 & 0.008 & Gm13986 & -2.48 & 0.019 \\
\hline Alpk3 & -6.59 & 0.009 & Sly & -6.59 & 0.009 & Gm14302 & -6.28 & 0.016 \\
\hline Bhlha15 & -3.52 & 0.027 & Smim24 & -3.65 & 0.022 & Gm14673 & -2.43 & 0.018 \\
\hline Bmp8b & -1.82 & 0.039 & Spata18 & -5.87 & 0.048 & Gm14886 & -6.02 & 0.033 \\
\hline Brs3 & 2.80 & 0.034 & Tfap2a & -2.26 & 0.033 & Gm15174 & -2.49 & 0.036 \\
\hline C6 & -6.15 & 0.023 & Tnfsf15 & -5.87 & 0.048 & Gm15302 & -5.87 & 0.048 \\
\hline C87414 & -5.87 & 0.048 & Uncx & -2.43 & 0.041 & Gm15839 & -1.96 & 0.047 \\
\hline Capn11 & -2.98 & 0.010 & Usp17la & -1.86 & 0.039 & Gm16028 & -6.15 & 0.023 \\
\hline Ccr1 & 3.72 & 0.046 & Vax2os & -2.15 & 0.017 & Gm16060 & -5.87 & 0.048 \\
\hline Ccr4 & -3.05 & 0.008 & Zfp345 & -2.89 & 0.032 & Gm16513 & -5.87 & 0.048 \\
\hline Cd200r3 & -1.97 & 0.040 & 1700007P06Rik & -6.02 & 0.033 & Gm17085 & -3.94 & 0.011 \\
\hline $\mathrm{Cd} 274$ & -1.80 & 0.037 & 1700021A07Rik & -3.38 & 0.042 & Gm17535 & -7.89 & 5.53E-09 \\
\hline Cd300lg & -3.58 & 0.005 & 1700128F08Rik & -2.23 & 0.012 & Gm20505 & -2.04 & 0.046 \\
\hline Ces5a & -1.83 & 0.034 & 1810019N24Rik & -6.02 & 0.033 & Gm20663 & -1.98 & 0.044 \\
\hline Cpa4 & -5.87 & 0.048 & 2810404F17Rik & -4.30 & 4.67E-04 & Gm20831 & -6.69 & 0.006 \\
\hline Csprs & -3.38 & 0.042 & $3110067 C 02$ Rik & -5.87 & 0.048 & Gm21292 & -3.39 & 0.003 \\
\hline Dsg3 & -2.23 & 0.022 & 4931406B18Rik & -1.69 & 0.050 & Gm2165 & -2.71 & 0.020 \\
\hline Egfros & -6.39 & 0.016 & 9530056K15Rik & -5.87 & 0.048 & Gm21719 & -6.15 & 0.023 \\
\hline Esp6 & -6.86 & 0.005 & C230088H06Rik & -4.17 & 0.005 & Gm21738 & -6.61 & 2.23E-09 \\
\hline Ffar2 & -3.38 & 0.042 & C430042M11Rik & -3.06 & 0.002 & Gm21776 & -6.69 & 0.006 \\
\hline Gcm1 & -2.38 & 0.013 & G430049J08Rik & -2.29 & 0.025 & Gm21784 & -3.77 & 0.015 \\
\hline Hopxos & -5.87 & 0.048 & CH36-246D16.4 & -3.02 & 0.009 & Gm21989 & 6.03 & 0.048 \\
\hline Hoxd11 & -3.61 & $3.48 \mathrm{E}-04$ & $\mathrm{CH} 36-35 \mathrm{H} 7.2$ & -7.54 & 0.001 & Gm23897 & -2.55 & 0.009 \\
\hline Insrr & -2.45 & 0.010 & CH36-399D20.1 & -7.93 & $1.73 \mathrm{E}-04$ & Gm26573 & -7.72 & $3.41 \mathrm{E}-04$ \\
\hline Itgb6 & -3.28 & 0.001 & Gm10038 & -2.16 & 0.026 & Gm26583 & -6.15 & 0.023 \\
\hline Klk14 & -3.48 & 0.002 & Gm10132 & -6.02 & 0.033 & Gm26648 & -6.28 & 0.016 \\
\hline Lmx1b & -3.02 & 0.003 & Gm10134 & -1.96 & 0.036 & Gm26704 & -5.05 & 9.17E-06 \\
\hline Lypd8 & -4.17 & 0.005 & Gm10172 & -2.31 & 0.013 & Gm26705 & -2.22 & 0.017 \\
\hline Mep1b & -2.76 & 0.020 & Gm10715 & -6.88 & 1.29E-09 & Gm26719 & -2.84 & 0.015 \\
\hline Mid1 & -1.78 & 0.045 & Gm10717 & -6.11 & 3.59E-09 & Gm26763 & -6.15 & 0.023 \\
\hline Mpz & -5.87 & 0.048 & Gm10718 & -6.15 & 2.98E-09 & Gm26804 & -7.01 & 0.003 \\
\hline Ms4a4b & -7.01 & 0.003 & Gm10719 & -6.56 & $5.50 \mathrm{E}-10$ & Gm26857 & -2.14 & 0.022 \\
\hline Muc19 & -2.46 & 0.011 & Gm10720 & -4.94 & $1.06 \mathrm{E}-06$ & Gm26870 & -6.03 & 4.96E-09 \\
\hline Muc6 & -5.87 & 0.048 & Gm10721 & -8.04 & $1.26 \mathrm{E}-04$ & Gm27956 & -6.39 & 0.016 \\
\hline Mylk4 & -2.85 & 0.002 & Gm10722 & -5.19 & 7.91E-07 & Gm3755 & -1.96 & 0.036 \\
\hline Nkapl & -2.72 & 0.046 & Gm10800 & -6.03 & 4.95E-09 & RP23-184B11.4 & -6.28 & 0.016 \\
\hline$N k \times 2-1$ & -3.40 & $2.13 \mathrm{E}-04$ & Gm10801 & -5.91 & 8.73E-09 & RP23-315H12.7 & -5.87 & 0.048 \\
\hline Orly & -6.69 & 0.006 & Gm11168 & -5.67 & $3.66 \mathrm{E}-08$ & RP23-458B6.16 & -6.86 & 0.005 \\
\hline Otc & 2.70 & 0.032 & Gm11231 & -2.18 & 0.032 & RP23-91 L14.2 & -6.59 & 0.009 \\
\hline Patl2 & -5.87 & 0.048 & Gm11398 & -5.87 & 0.048 & RP24-112B7.3 & -3.24 & 0.012 \\
\hline Pcdh12 & 2.83 & 0.046 & Gm11883 & -5.87 & 0.048 & RP24-209E1.3 & -6.02 & 0.033 \\
\hline Pou1f1 & -2.62 & 0.003 & Gm11948 & -5.87 & 0.048 & RP24-228|22.1 & -6.69 & 0.006 \\
\hline Pou2f2 & -1.83 & 0.043 & Gm12177 & -2.43 & 0.021 & RP24-319B23.2 & -4.68 & 0.001 \\
\hline
\end{tabular}


Table 3 Differentially expressed genes in the brain of HFD-fed mice (Continued)

\begin{tabular}{|c|c|c|c|c|c|c|c|c|}
\hline Pou2f3-rs1 & -6.02 & 0.033 & Gm12496 & -2.91 & 0.013 & RP24-369 J17.1 & -6.39 & 0.016 \\
\hline Psg28 & -1.83 & 0.042 & Gm12652 & -6.28 & 0.016 & RP24-446E18.2 & -6.02 & 0.033 \\
\hline Rbp2 & -3.72 & 0.018 & Gm13086 & -2.34 & 0.023 & RP24-482E11.1 & -5.87 & 0.048 \\
\hline Rdh19 & -6.28 & 0.016 & Gm13465 & -2.54 & 0.033 & RP24-72B9.10 & -6.02 & 0.033 \\
\hline Rhox8 & -6.15 & 0.023 & Gm13691 & -6.28 & 0.016 & & & \\
\hline Serpina10 & -4.52 & 0.002 & Gm13961 & -5.87 & 0.048 & & & \\
\hline
\end{tabular}

$F C$ fold change

(Ighe) and immunoglobulin heavy constant gamma 1 (G1m marker) (Ighg1) were increased to 52.71- and 6.02-fold respectively, while the mRNA levels of chemokine (C-C motif) receptor 9 (Ccr9), CD8 antigen, alpha chain $(\mathrm{Cd} 8 \mathrm{a})$, recombination activating gene $1(\operatorname{Rag} 1)$, recombination activating gene 2 (Rag2) and suppression inducing transmembrane adaptor 1 (Sit1) were decreased to 6.32-, 3.94-, 512-, 19.70- and 5.10- fold respectively. Circulatory system process was also significantly changed in the lung of HFD-fed mice, with the down-regulation of natriuretic peptide precursor $\mathrm{A}$ (Nppa) (7.78-fold) and natriuretic peptide precursor B (Nppb) (21.26-fold). Leptin (Lep) involved in the regulation of cholesterol absorption were increased to 5.70fold and CART prepropeptide (Cartpt) related to cell glucose homeostasis were decreased to 62.25 -fold. Other overrepresented biological processes included circadian regulation of gene expression and positive regulation of transmission of nerve impulse.

\section{Verification of RNA-seq data}

qRT-PCR was used to validate the expression levels measured by RNA-seq for 22 selected genes ( 8 genes in the brain, 9 genes in the spleen and 5 genes in the lung) from the list of differently expressed genes. As demonstrated in Fig. 4, qRT-PCR showed significant alterations in the expression of the 17 genes in correspondence with the findings from the RNA-sequencing analysis, while no obvious differential expression was detected for the 5 genes of Trap2a, Cd274, Ighv1-84, Igkv5-39 and Traj37 by qRT-PCR.

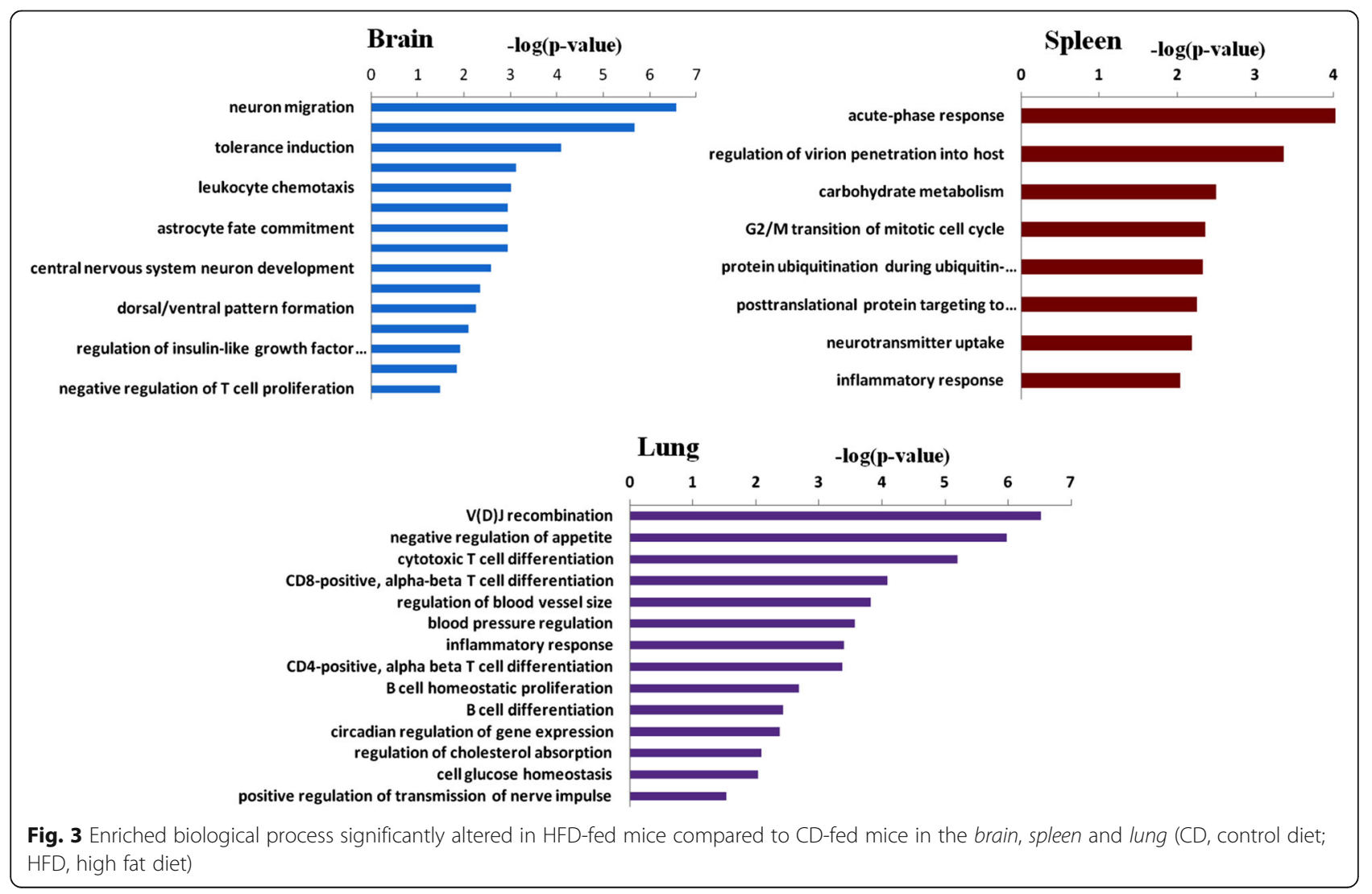


Table 4 Differentially expressed genes in the spleen of HFD-fed mice

\begin{tabular}{|c|c|c|c|c|c|}
\hline Gene symbol & $\log _{2} \mathrm{FC}$ & $P$-value & Gene symbol & $\log _{2} \mathrm{FC}$ & $P$-value \\
\hline Acr & -6.09 & 0.033 & 5330434G04Rik & -5.77 & 0.048 \\
\hline Ccdc38 & -6.09 & 0.033 & 5730596B20Rik & -5.77 & 0.048 \\
\hline Cngb3 & -5.77 & 0.048 & AY036118 & -6.09 & 0.033 \\
\hline Fau-ps2 & 2.73 & 0.027 & BC061212 & -3.01 & 0.046 \\
\hline Fcrls & -6.22 & 0.023 & BC068157 & 5.92 & 0.048 \\
\hline Folr2 & -6.22 & 0.023 & D830025C05Rik & -5.94 & 0.033 \\
\hline Frmpd4 & -6.35 & 0.016 & Gm10071 & -5.77 & 0.048 \\
\hline Hs3st5 & -5.77 & 0.048 & Gm11455 & 2.26 & 0.047 \\
\hline Ighv1-84 & -3.54 & $2.38 \mathrm{E}-04$ & Gm11800 & -3.62 & 0.010 \\
\hline lgkj1 & -5.77 & 0.048 & Gm11957 & 6.33 & 0.023 \\
\hline lgkj4 & 3.06 & 0.021 & Gm12763 & -2.40 & 0.034 \\
\hline Igkv5-39 & -1.85 & 0.034 & Gm13483 & -3.01 & 0.046 \\
\hline $\operatorname{Ln} \times 1$ & -5.94 & 0.033 & Gm14444 & -6.09 & 0.033 \\
\hline Lrrc7 & -2.83 & 0.046 & Gm15302 & -6.22 & 0.023 \\
\hline Mcpt4 & -3.01 & 0.046 & Gm15785 & -5.94 & 0.033 \\
\hline$m t-T m$ & -5.77 & 0.048 & Gm17305 & -5.94 & 0.033 \\
\hline Nek10 & -5.94 & 0.033 & Gm20544 & -3.01 & 0.046 \\
\hline Palm2Akap2 & -3.21 & 0.027 & Gm21719 & -3.30 & 0.027 \\
\hline Pcdhb10 & -6.09 & 0.033 & Gm2237 & -5.94 & 0.033 \\
\hline Reg2 & -3.33 & $2.65 \mathrm{E}-04$ & Gm24436 & 6.33 & 0.023 \\
\hline Reg3a & -4.03 & $1.50 \mathrm{E}-04$ & Gm25153 & 2.80 & 0.035 \\
\hline Reg3b & -3.02 & 0.001 & Gm25931 & 2.65 & 0.046 \\
\hline Rpl9-ps3 & -5.94 & 0.033 & Gm26619 & -2.95 & 0.008 \\
\hline Slc17a6 & -5.77 & 0.048 & Gm26807 & -6.09 & 0.033 \\
\hline St6gal2 & -5.77 & 0.048 & Gm26825 & -2.37 & 0.029 \\
\hline Tceal3 & -3.01 & 0.046 & Gm6136 & -6.22 & 0.023 \\
\hline Tpd52l1 & -5.77 & 0.048 & Gm6612 & 2.65 & 0.046 \\
\hline Traj37 & 6.14 & 0.033 & RP23-446G23.1 & 2.80 & 0.035 \\
\hline Vat1l & -3.01 & 0.046 & RP24-369 J17.1 & -2.89 & 0.039 \\
\hline Zfp42 & -5.77 & 0.048 & RP24-44H8.4 & -2.86 & 0.011 \\
\hline 1700095A21Rik & -5.94 & 0.033 & & & \\
\hline
\end{tabular}

FC fold change

\section{Discussion}

Consistent with previous reports $[6,16]$, our results showed that the consumption of a high-fat diet for 3 days significantly increased plasma glucose level. The high-fat diet also increased plasma cholesterol and HDL cholesterol concentrations. Plasma triglycerides concentration decreased significantly after short-term high-fat feeding. Indeed, decreased triglycerides level was previously reported as early as 3 days after beginning a high-fat diet in a study that involved mice fed a chronic high-fat diet [17]. A randomized, double-blind, crossover study in 12 healthy subjects reported that plasma triglycerides concentration was significantly lower after a 3-d high-fat
Table 5 Differentially expressed genes in the lung of HFD-fed mice

\begin{tabular}{|c|c|c|c|c|c|}
\hline Gene symbol & $\log _{2} \mathrm{FC}$ & $P$-value & Gene symbol & $\log _{2} \mathrm{FC}$ & $P$-value \\
\hline Abcc6 & 5.72 & 0.048 & Satb1 & -1.99 & 0.022 \\
\hline Arpp21 & -5.29 & 7.83E-07 & Scn1a & -2.97 & 0.042 \\
\hline Arsi & -2.28 & 0.034 & Sit1 & -2.35 & 0.023 \\
\hline Bmp10 & -4.72 & 4.94E-06 & Skint10 & -5.96 & 0.033 \\
\hline Cartpt & -5.96 & 0.033 & Spo11 & -3.46 & 0.001 \\
\hline Casp14 & -5.96 & 0.033 & St18 & 5.72 & 0.048 \\
\hline Ccr9 & -2.66 & 0.004 & Syt13 & -2.12 & 0.039 \\
\hline Cd8a & -1.98 & 0.025 & Syt2 & -3.54 & 0.035 \\
\hline Cdca5 & -2.28 & 0.036 & Tdrd5 & -2.93 & 0.006 \\
\hline Chrna9 & -4.53 & 0.003 & Tgm5 & -2.78 & 0.039 \\
\hline Crisp1 & -1.87 & 0.040 & Themis & -2.26 & 0.010 \\
\hline Dntt & -7.36 & $2.18 \mathrm{E}-09$ & Tnni1 & 6.36 & 0.016 \\
\hline Elovl3 & -1.96 & 0.036 & Trbc1 & -1.97 & 0.025 \\
\hline Epyc & -6.15 & 0.023 & Trbv17 & -6.32 & 0.016 \\
\hline Gucy2g & 5.91 & 0.048 & Trbv4 & -3.66 & 0.027 \\
\hline Hist1h1a & -1.88 & 0.034 & Trim10 & 1.90 & 0.040 \\
\hline Ighe & 5.72 & 0.048 & Tube1 & -1.96 & 0.050 \\
\hline lghg1 & 2.59 & 0.011 & Ubd & -3.06 & 0.017 \\
\hline Ighv11-1 & -2.90 & 0.002 & Vmn2r96 & -3.54 & 0.035 \\
\hline Ighv11-2 & -3.20 & 0.001 & Xkrx & -2.35 & 0.008 \\
\hline lghv12-3 & -3.46 & 0.001 & Xlr5a & 5.72 & 0.048 \\
\hline Ighv1-53 & 1.92 & 0.046 & Zan & 3.20 & 0.046 \\
\hline lghv1-54 & 2.42 & 0.031 & 1600029015Rik & 5.91 & 0.048 \\
\hline lghv1-84 & -2.28 & 0.036 & 4930455G09Rik & -3.66 & 0.027 \\
\hline lghv7-3 & -2.04 & 0.041 & 9330132A10Rik & 6.07 & 0.033 \\
\hline Ighv7-4 & -6.47 & 0.012 & BB031773 & -7.46 & 0.001 \\
\hline lgkv14-126 & -2.80 & 0.002 & BC028471 & -2.85 & 0.027 \\
\hline lgkv1-99 & 6.89 & 0.004 & BC065403 & -5.96 & 0.033 \\
\hline lgkv3-2 & 1.95 & 0.039 & Gm10489 & 5.72 & 0.048 \\
\hline lgkv4-91 & -2.95 & 0.002 & Gm10715 & 5.91 & 0.048 \\
\hline Insc & 5.91 & 0.048 & Gm10717 & 1.74 & 0.045 \\
\hline Lctl & 2.40 & 0.031 & Gm10785 & 5.91 & 0.048 \\
\hline Lep & 2.51 & 0.008 & Gm13855 & 5.72 & 0.048 \\
\hline Lin28a & 5.72 & 0.048 & Gm15340 & -6.47 & 0.012 \\
\hline Ltb4r1 & 2.93 & 0.022 & Gm15405 & -2.16 & 0.045 \\
\hline $\mathrm{mt}-\mathrm{Tt}$ & 6.22 & 0.023 & Gm15576 & -5.96 & 0.033 \\
\hline Nppa & -2.96 & 0.001 & Gm20438 & 5.91 & 0.048 \\
\hline Nppb & -4.41 & $4.38 \mathrm{E}-05$ & Gm26202 & 6.07 & 0.033 \\
\hline Prom2 & -2.66 & 0.031 & Gm26316 & 5.72 & 0.048 \\
\hline Prss16 & -9.54 & 4.44E-07 & Gm26870 & 2.00 & 0.021 \\
\hline Rag1 & -9.00 & $2.01 \mathrm{E}-12$ & RP23-230A14.1 & 5.72 & 0.048 \\
\hline Rag2 & -4.30 & $3.9 \mathrm{E}-04$ & & & \\
\hline
\end{tabular}

FC fold change 


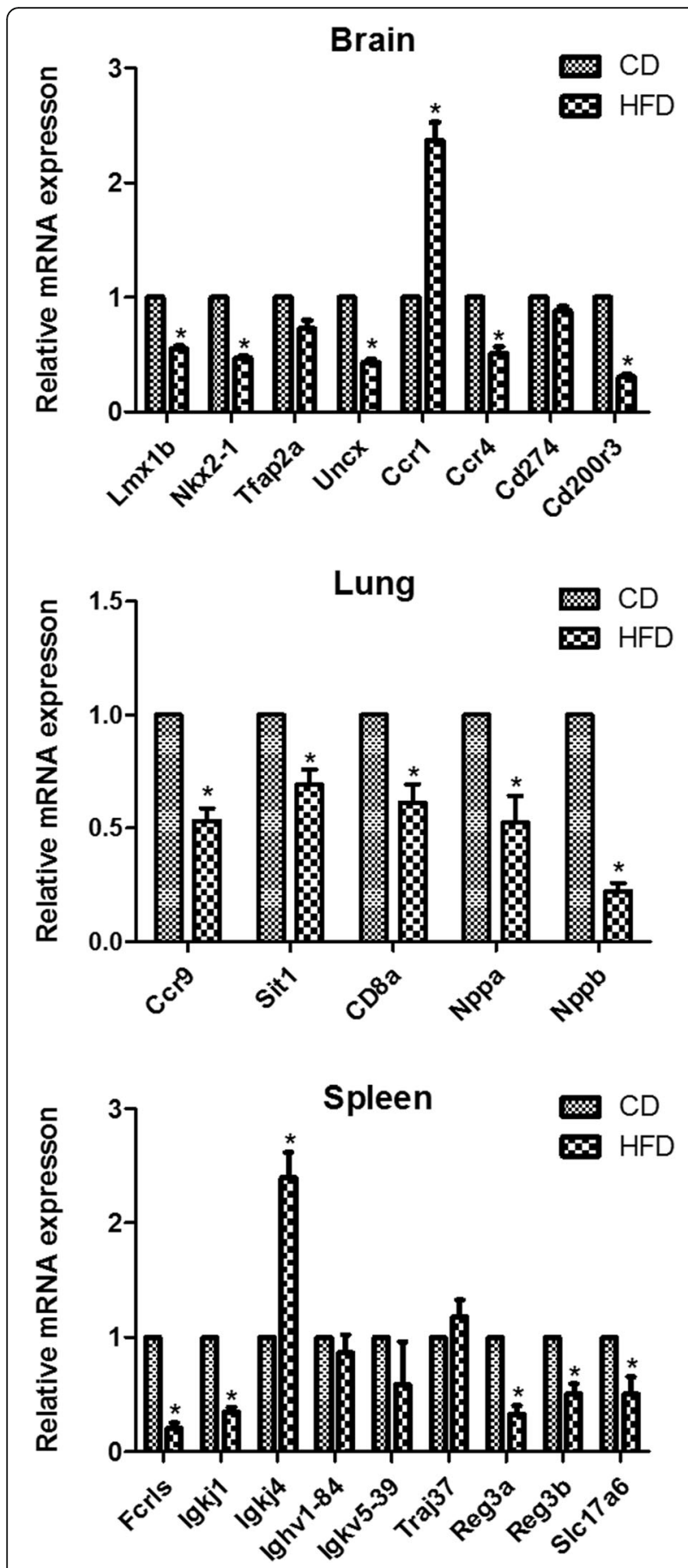

Fig. 4 Confirmation of differential gene expression via qRT-PCR analysis (CD, control diet; HFD, high fat diet; ${ }^{*} P<0.05$ vs. CD group). The expression value was normalized to the $\beta$-actin expression level. Values are expressed as average mRNA expression \pm SEM bars

diet [18]. The low plasma triglycerides could be due to increased liver triglyceride content, possibly resulting in triglycerides being stored in the liver [19].

To our knowledge, we first reported that 3 days of high-fat feeding can induce exercise performance decrease in mice prior to substantial weight gain. In contrast to previous studies which conducted microarray analysis in a single tissue [6-9], we used RNA-seq to investigate the global gene expression profiles of ten tissues in the early response to fat intake. Interestingly, our study showed that differential gene expression patterns existed in the ten tissues. Previous researches on the effect of HFD mainly focused on the liver, skeletal muscle, intestine and heart, which were thought to have significant responses to consumption of high fat diets. However, in our results, the brain, spleen and lung were demonstrated to have more pronounced transcriptional changes than other tissues following 3 days of high-fat feeding. The simultaneous analysis of multi-tissues by RNA sequencing yielded information which had not been revealed by previous analyses of a single tissue.

In the brain of HFD-fed mice, dopamine neurons differentiation related genes including Lmx1b, Nkx2-1 and Uncx were found to be significantly down-regulated. Lmx1b, a key transcription factor for the specification of dopaminergic cell fate, has been reported to increase midbrain size and allocation of dopamine progenitors by promoting Wnt1/Wnt signaling [20]. Deficiency of Nkx2-1 in mice would lead to a remarkable abnormality in the trajectory of the ascending dopamine pathway [21]. Uncx, also known as Uncx4.1, is involved in the development of midbrain dopaminergic neurons [22]. Therefore, the down-regulation of these genes may contribute to impaired exercise capacity, which is supported by the observation of a link between dopamine and levels of physical exercise [23]. Moreover, Lmx1b and Nkx2-1 were demonstrated to regulate the migration of the superficial dorsal horn neurons and interneurons to the striatum or cortex, respectively [24, 25]. The decreased expression of the two genes suggested disturbed neuronal migration in HFD-fed mice, which may result in abnormal development of the nervous system. Inflammation is considered to be one of the important factors for deterioration of physical performance [26]. Similarly, our results also suggested that many immune-related genes were significantly altered in the brain of HFD-fed mice. Ccr1, which involved in the host response to pathogens and several inflammatory conditions [27], were significantly up-regulated. On the other hand, Ccr4 and Cd200r3 showed decreased expression in HFD-fed mice. Ccr4 were found to be functionally expressed on peripheral blood $\mathrm{CD} 4{ }^{+} \mathrm{CD} 25^{+}$regulatory $\mathrm{T}$ (Treg) cells [28] CD200 imparts an immunoregulatory signal through the receptor for CD200, leading to the suppression of $\mathrm{T}$ cell-mediated immune responses [29]. The upregulation of Ccr1, coupled with the down-regulation of Ccr4 and Cd200r3, suggested the up-regulation of an inflammatory response toward the high fat diet in the brain. Consistently, it was reported that consumption of 
a HFD with 1 to 3 days induced hypothalamic inflammatory in both rats and mice [30]. However, the genes related to hypothalamic inflammatory signaling in the above study were not found to be significantly altered in our results. This discrepancy may reflect a difference between specific brain region and the whole brain.

The immune system has been considered to be affected by HFD exposure over a period of several weeks [31]. However, in the present study, we found that 3 days of HFD feeding had induced disturbed immune response in spleen, which is a major organ involved in B-cell maturation. Fcrls, which belongs to Fc receptor-like family possessing inhibitory and/or activating signaling motifs in B cell differentiation [32], were significantly downregulated in response to HFD feeding. Similarly, microarray profiling carried out by Cui et al. in the spleen of C57BL/6 mice fed on a HFD also showed a decreased expression of Fc receptor [33]. Meanwhile, we observed abnormal expression of a few genes involved in immunoglobulin/B cell receptor signaling. Igkj4 showed significant up-regulation in HFD-fed mice, whereas the expression of Igkj1 were significantly decreased. Additionally, Reg3a and Reg3b, which play critical roles in acute-phase response [34, 35], were significantly downregulated. The findings suggested that immune dysfunction was implicated in the spleen in response to 3 days of HFD feeding. Interestingly, neurotransmitter uptake related genes were significantly changed, such as solute carrier family 17 (sodium-dependent inorganic phosphate cotransporter), member $6($ Slc17a6) which plays a key role in the transport of glutamate into synaptic vesicles before exocytotic release and the regulation of glutamate signaling [36].

Evidence has increasingly shown that a HFD regarded as a primary cause for numerous diseases including diabetes, hypertension, and steatohepatitis. However, few studies have been carried out to examine the effect of a HFD on the lung. To our surprise, the PCA results showed pronounced transcriptional changes in the lung and this is the first report to investigate the lung transcriptome profile after 3 days of HFD feeding. As the lung is a major site of immune regulation, our results revealed that many immune-related genes were significantly altered. Chemokine receptor Ccr9, which have proved to be important in the Treg cells mediated self-tolerance [37], was markedly down-regulated in HFD-fed mice. Sit1, a critical negative regulator of TCR-mediated signaling [38], showed significant down-regulation as well. The decreased expression of $\mathrm{Ccr} 9$ and Sit1 suggested an activated inflammatory response affected by HFD in the lung, which was in agreement with the previous study of the involvement of a HFD on lung inflammation [39]. In addition, we discovered decreased expression of CD8a in HFD-fed mice, which is important in cell-mediated immune defense and
T-cell development [40]. Moreover, genes involved in T cell receptor signaling and immunoglobulin/ B cell receptor signaling were found to be significant altered. This study also revealed that circulatory system related genes were down-regulated by HFD in the lung. Nppa and Nppb are the precursor of atrial natriuretic peptide (ANP) and b-type natriuretic peptide (BNP), which have important physiological functions in the regulation of vascular tone and plasma volume [41]. ANP exhibits a protective role in the lung function in acute lung injury apart from its vasodilatory and natriuretic effects [42]. Intake of a HFD had been proved to make a slower pulmonary $\mathrm{O}_{2}$ uptake kinetics and attenuate microvascular blood flow and $\mathrm{O}_{2}$ delivery during the transition to moderate intensity exercise [43]. Therefore, we speculated that the decreased expression of Nppa and Nppb may contribute to the impaired exercise capacity in HFD-fed mice.

\section{Conclusions}

The simultaneous analysis of ten tissues following 3 days of high-fat feeding by RNA-seq technology revealed that the brain, spleen and lung had more pronounced transcriptional changes than other tissues. Dysregulation of peripheral and central immune response had been implicated in the early stage of the response to HFD exposure. Neurotransmission-related genes and circulatory system process related genes were markedly downregulated in the brain and lung, respectively. These findings provide new insights for the deleterious effects of a HFD and contribute to the understanding of molecular mechanisms of exercise performance decrease induced by short-term high-fat feeding.

\section{Additional files}

Additional file 1: Table S1. List of Primer sequences used for qRT-PCR. (DOC $57 \mathrm{~kb}$ )

Additional file 2: Table S2. Plasma biochemical parameters of CD-fed and HFD-fed mice. (DOCX $21 \mathrm{~kb}$ )

\footnotetext{
Abbreviations

ALB: Albumin; ALP: Alkaline phosphatase; ALT: Alanine aminotransferase; ANP: Atrial natriuretic peptide; ApoE: Apolipoprotein E; AST: Aspartate aminotransferase; BNP: b-type natriuretic peptide; Ca: Calcium; CD: Control diet; CK: Creatine kinase; Cl: Chlorine; CPM: Counts per million; $\mathrm{Cr}$ : Creatinine; CRP: C-reactive protein; CysC: Cystatin C; DBIL: Direct bilirubin; DEGs: Differentially expressed genes; $\mathrm{HBDH}$ : A-hydroxybutyric acid dehydrogenase; HCY: Homocysteine; HDL-C: High-density lipoproteincholesterol; HFD: High fat diet; IBIL: Indirect bilirubin; K: Potassium;

LDH: Lactate dehydrogenas; LDL-C: Low-density lipoprotein-cholesterol; MAS: Molecule annotation system; Na: Sodium; PCA: Principle component analysis; RNA-seq: RNA sequencing; RPKM: Reads Per Kilobase of exon model per Million mapped reads; SOD: Superoxide dismutase; TBA: Total bile acid; TBIL: Total bilirubin; TP: Total protein; Treg: Regulatory T; UA: Uric acid
}

Acknowledgements

Not applicable. 


\section{Funding}

This work was supported by the National Science Foundation of China (Nos. 81373707, 81403447 and 81603520), the Natural Science Foundation of Guangdong Province, China (Nos. 2014A030313292, 2014A030310072 and 2016A030310084), the Science \& Technical Plan of Guangzhou, Guangdong, China (No. 2014Y2-00504), the Administration of Traditional Medicine of Guangdong province (No. 20161063), the Fundamental Research Funds for the Central Universities (No. 21616315) and the Special Funds for the Cultivation of Guangdong College Students Scientific and Technological Innovation (No. pdjh2016b0093).

\section{Availability of data and materials}

All data generated or analysed during this study are included in this published article and its Additional file 1: Table S1 and Additional file 2: Table S2.

\section{Authors' contributions}

$X Z$ and $Y L$ conceived and designed the experiments. YX, WW, JC and PJ acquired the data. $X F, X N$ and $L C$ analyzed and interpreted the data. $Y X, W W$ and LC drafted the manuscript. $X Z$ and HK revised the manuscript for important intellectual content. $X Z$ and $Y L$ supervised the study. All authors were involved in the formulation of the research questions. All authors read and approved the final manuscript.

\section{Competing interests}

The authors declare that they have no competing interests.

\section{Consent for publication}

Not applicable.

\section{Ethics approval}

Animal experiments were approved by the Animal Care and Use Committee of Southern Medical University (Approval No.2013027).

\section{Author details}

${ }^{1}$ Department of Traditional Chinese Medicine, School of Medicine, Jinan University, Guangzhou, China. ${ }^{2}$ School of Traditional Chinese Medicine, Southern Medical University, Guangzhou, China. ${ }^{3}$ Experimental Animal Center, Southern Medical University, Guangzhou, China. ${ }^{4}$ School of Chinese Medicine, Hong Kong Baptist University, Hong Kong, China.

Received: 14 July 2016 Accepted: 25 January 2017

\section{Published online: 02 February 2017}

\section{References}

1. Riccardi G, Giacco R, Rivellese AA. Dietary fat, insulin sensitivity and the metabolic syndrome. Clin Nutr. 2004;23:447-56.

2. Miller WC, Bryce GR, Conlee RK. Adaptations to a high-fat diet that increase exercise endurance in male rats. J Appl Physiol Respir Environ Exerc Physiol. 1984;56:78-83

3. Simi B, Sempore B, Mayet MH, Favier RJ. Additive effects of training and high-fat diet on energy metabolism during exercise. J Appl Physiol. 1991;71:197-203.

4. Lapachet RA, Miller WC, Arnall DA. Body fat and exercise endurance in trained rats adapted to a high-fat and/or high-carbohydrate diet. J Appl Physiol. 1996;80:1173-9.

5. Murray AJ, Knight NS, Cochlin LE, McAleese S, Deacon RM, Rawlins JN, et al. Deterioration of physical performance and cognitive function in rats with short-term high-fat feeding. FASEB J. 2009;23:4353-60.

6. De Giorgio MR, Yoshioka M, St-Amand J. Feeding induced changes in the hypothalamic transcriptome. Clin Chim Acta. 2009:406:103-7.

7. Yoshioka M, Bolduc C, Raymond V, St-Amand J. High-fat meal-induced changes in the duodenum mucosa transcriptome. Obesity (Silver Spring). 2008;16:2302-7.

8. de Wilde J, Mohren R, van den Berg S, Boekschoten M, Dijk KW, de Groot P, et al. Short-term high fat-feeding results in morphological and metabolic adaptations in the skeletal muscle of C57BL/6 J mice. Physiol Genomics. 2008:32:360-9.

9. Nishikawa S, Sugimoto J, Okada M, Sakairi T, Takagi S. Gene expression in livers of BALB/C and C57BL/6 J mice fed a high-fat diet. Toxicol Pathol. 2012:40:71-82

10. HP T, Ariyurek Y, Thygesen HH, Vreugdenhil E, Vossen RH, de Menezes RX, et al. Deep sequencing-based expression analysis shows major advances in robustness, resolution and inter-lab portability over five microarray platforms. Nucleic Acids Res. 2008;36:e141.

11. Wang SY, Huang WC, Liu CC, Wang MF, Ho CS, Huang WP, et al. Pumpkin (Cucurbita moschata) fruit extract improves physical fatigue and exercise performance in mice. Molecules. 2012;17:11864-76.

12. Wu RM, Sun YY, Zhou TT, Zhu ZY, Zhuang JJ, Tang $X$, et al. Arctigenin enhances swimming endurance of sedentary rats partially by regulation of antioxidant pathways. Acta Pharmacol Sin. 2014;35:1274-84.

13. Tan W, Yu KQ, Liu YY, Ouyang MZ, Yan MH, Luo R, et al. Anti-fatigue activity of polysaccharides extract from Radix Rehmanniae Preparata. Int J Biol Macromol. 2012;50:59-62.

14. Robinson MD, McCarthy DJ, Smyth GK. edger: a Bioconductor package for differential expression analysis of digital gene expression data. Bioinformatics. 2010;26:139-40.

15. Wang L, Sun L, Huang J, Jiang M. Cyclin-dependent kinase inhibitor 3 (CDKN3) novel cell cycle computational network between human nonmalignancy associated hepatitis/cirrhosis and hepatocellular carcinoma (HCC) transformation. Cell Prolif. 2011;44:291-9.

16. Lee YS, Li P, Huh JY, Hwang IJ, Lu M, Kim JI, et al. Inflammation is necessary for long-term but not short-term high-fat diet-induced insulin resistance. Diabetes. 2011:60:2474-83.

17. Petit V, Arnould L, Martin P, Monnot MC, Pineau T, Besnard P, et al. Chronic high-fat diet affects intestinal fat absorption and postprandial triglyceride levels in the mouse. J Lipid Res. 2007;48:278-87.

18. Tremblay AJ, Lamarche B, Guay V, Charest A, Lemelin V, Couture P. Short-term, high-fat diet increases the expression of key intestinal genes involved in lipoprotein metabolism in healthy men. Am J Clin Nutr. 2013:98:32-41.

19. Jin ES, Beddow SA, Malloy CR, Samuel VT. Hepatic glucose production pathways after three days of a high-fat diet. Metabolism. 2013:62:152-62.

20. Anderegg A, Lin HP, Chen JA, Caronia-Brown G, Cherepanova N, Yun B, et al. An Lmx1b-miR135a2 regulatory circuit modulates Wnt1/Wnt signaling and determines the size of the midbrain dopaminergic progenitor pool. PLoS Genet. 2013:9:e1003973.

21. Kawano H, Horie M, Honma S, Kawamura K, Takeuchi K, Kimura S. Aberrant trajectory of ascending dopaminergic pathway in mice lacking Nkx2.1. Exp Neurol. 2003;182:103-12.

22. Rabe TI, Griesel G, Blanke S, Kispert A, Leitges M, van der Zwaag B, et al. The transcription factor Uncx4.1 acts in a short window of midbrain dopaminergic neuron differentiation. Neural Dev. 2012;7:39.

23. Mathes WF, Nehrenberg DL, Gordon R, Hua K, Garland TJ, Pomp D, et al Dopaminergic dysregulation in mice selectively bred for excessive exercise or obesity. Behav Brain Res. 2010;210:155-63.

24. Ding YQ, Yin J, Kania A, Zhao ZQ, Johnson RL, Chen ZF, et al. Lm×1b controls the differentiation and migration of the superficial dorsal horn neurons of the spinal cord. Development. 2004;131:3693-703.

25. Elias $L A$, Potter $G B$, Kriegstein AR. A time and a place for $n k \times 2-1$ in interneuron specification and migration. Neuron. 2008;59:679-82.

26. Katafuchi T, Kondo T, Take S, Yoshimura M. Enhanced expression of brain interferon-alpha and serotonin transporter in immunologically induced fatigue in rats. Eur J Neurosci. 2005;22:2817-26.

27. Rossi D, Zlotnik A. The biology of chemokines and their receptors. Annu Rev Immunol. 2000;18:217-42.

28. lellem A, Mariani M, Lang R, Recalde H, Panina-Bordignon P, Sinigaglia F, et al. Unique chemotactic response profile and specific expression of chemokine receptors CCR4 and CCR8 by CD4(+)CD25(+) regulatory T cells. J Exp Med. 2001;194:847-53.

29. Rosenblum MD, Olasz E, Woodliff JE, Johnson BD, Konkol MC, Gerber KA, et al. CD200 is a novel p53-target gene involved in apoptosis-associated immune tolerance. Blood. 2004:103:2691-8.

30. Thaler JP, Yi CX, Schur EA, Guyenet SJ, Hwang BH, Dietrich MO, et al. Obesity is associated with hypothalamic injury in rodents and humans. J Clin Invest. 2012;122:153-62.

31. Miyazaki $Y$, Iwabuchi $K$, Iwata D, Miyazaki A, Kon $Y$, Niino $M$, et al. Effect of high fat diet on NKT cell function and NKT cell-mediated regulation of Th1 responses. Scand J Immunol. 2008:67:230-7.

32. Davis RS, Stephan RP, Chen CC, Dennis GJ, Cooper MD. Differential B cell expression of mouse Fc receptor homologs. Int Immunol. 2004;16:1343-53.

33. Cui J, Xiao Y, Shi YH, Wang B, Le GW. Lipoic acid attenuates high-fat-dietinduced oxidative stress and B-cell-related immune depression. Nutrition. 2012;28:275-80 
34. Wang L, Huang J, Jiang M, Lin H. Tissue-specific transplantation antigen P35B (TSTA3) immune response-mediated metabolism coupling cell cycle to postreplication repair network in no-tumor hepatitis/cirrhotic tissues (HBV or HCV infection) by biocomputation. Immunol Res. 2012:52:258-68.

35. van Ampting MT, Loonen LM, Schonewille AJ, Konings I, Vink C, lovanna J, Chamaillard M, et al. Intestinally secreted C-type lectin Reg3b attenuates salmonellosis but not listeriosis in mice. Infect Immun. 2012;80:1115-20.

36. Brumovsky P, Watanabe M, Hokfelt T. Expression of the vesicular glutamate transporters- 1 and -2 in adult mouse dorsal root ganglia and spinal cord and their regulation by nerve injury. Neuroscience. 2007;147:469-90.

37. Wermers JD, McNamee EN, Wurbel MA. The chemokine receptor CCR9 is required for the T-cell-mediated regulation of chronic ileitis in mice. Gastroenterology. 2011;140:1526-35

38. Arndt B, Krieger T, Kalinski T, Thielitz A, Reinhold D, Roessner A, et al. The transmembrane adaptor protein SIT inhibits TCR-mediated signaling. PLoS One. 2011;6:e23761.

39. Naura AS, Hans CP, Zerfaoui M, Errami Y, Ju J, Kim H, et al. High-fat diet induces lung remodeling in ApoE-deficient mice: an association with an increase in circulatory and lung inflammatory factors. Lab Invest. 2009;89:1243-51.

40. Xu SW, Wu JY, Hu KS, Ping HL, Duan ZG, Zhang HF. Molecular cloning and expression of orange-spotted grouper (Epinephelus coioides) CD8alpha and CD8 beta genes. Fish Shellfish Immunol. 2011;30:600-8.

41. Baxter GF. Natriuretic peptides and myocardial ischaemia. Basic Res Cardiol. 2004;99:90-3.

42. Mitaka C, Hirata Y, Nagura T, Tsunoda Y, Amaha K. Beneficial effect of atrial natriuretic peptide on pulmonary gas exchange in patients with acute lung injury. Chest. 1998;1 14:223-8.

43. Raper JA, Love LK, Paterson DH, Peters SJ, Heigenhauser GJ, Kowalchuk JM. Effect of high-fat and high-carbohydrate diets on pulmonary $\mathrm{O} 2$ uptake kinetics during the transition to moderate-intensity exercise. J Appl Physiol. 2014:117:1371-9.

\section{Submit your next manuscript to BioMed Central and we will help you at every step:}

- We accept pre-submission inquiries

- Our selector tool helps you to find the most relevant journal

- We provide round the clock customer support

- Convenient online submission

- Thorough peer review

- Inclusion in PubMed and all major indexing services

- Maximum visibility for your research

Submit your manuscript at www.biomedcentral.com/submit

) Biomed Central 